\title{
Discussion of coupled and tripled coincidence point theorems for $\varphi$-contractive mappings without the mixed $g$-monotone property
}

\author{
Erdal Karapınar ${ }^{1,2}$, Antonio Roldán $^{3}$, Naseer Shahzad ${ }^{4 *}$ and Wutiphol Sintunavarat ${ }^{5}$
}

\section{"Correspondence:}

naseer_shahzad@hotmail.com; nshahzad@kau.edu.sa

${ }^{4}$ Department of Mathematics, King Abdulaziz University, P.O. Box 80203

Jeddah, 21589, Saudi Arabia

Full list of author information is

available at the end of the article

\begin{abstract}
After the appearance of Ran and Reuring's theorem and Nieto and Rodríguez-López's theorem, the field of fixed point theory applied to partially ordered metric spaces has attracted much attention. Coupled, tripled, quadrupled and multidimensional fixed point results has been presented in recent times. One of the most important hypotheses of these theorems was the mixed monotone property. The notion of invariant set was introduced in order to avoid the condition of mixed monotone property, and many statements have been proved using these hypotheses. In this paper we show that the invariant condition, together with transitivity, lets us to prove in many occasions similar theorems to which were introduced using the mixed monotone property.
\end{abstract}

MSC: 46T99; 47H10;47H09; 54H25

Keywords: partially ordered set; fixed point; contractive mapping; mixed monotone property; F-invariant set

\section{Introduction}

One of the core recent research topics in Fixed Point Theory is multidimensional fixed point results in the context of various abstract space. This trend was initiated by the well-known paper of Gnana-Bhaskar and Lakshmikantham [1]. Following this pioneering work, several authors reported various results in the setting of partially ordered metric spaces. Recently, the notion of coupled fixed point was extended to the higher dimensions by defining tripled, quadrupled and, hence, multidimensional fixed points [2-5]. In [6], Roldán et al. proved that most of the multidimensional fixed point results can be derived from the existing fixed point theorems in the context of partially preordered metric spaces with the additional hypothesis of the mixed monotone property. Meanwhile, in the literature, several multidimensional fixed point theorems have appeared in which the authors omitted the notion of mixed property by adding a weaker conditions such as F-closed, F-invariant, etc.

In this paper we will show that the notion of transitive $F$-closed (or $F$-invariant) set is equivalent to the concept of preordered set and, then some recent multidimensional results using $F$-invariant sets can be reduced to well-known results on partially ordered metric spaces.

O2014 Karapınar et al.: licensee Springer. This is an Open Access article distributed under the terms of the Creative Commons Attribution License (http://creativecommons.org/licenses/by/2.0), which permits unrestricted use, distribution, and reproduction in any medium, provided the original work is properly cited. 


\section{Preliminaries}

Henceforth, let $X$ be a nonempty set. Given a positive integer $n$, let $X^{n}$ be the product space $X \times X \times \stackrel{n}{ } \times X$. Let $\mathbb{N}=\{0,1,2, \ldots\}$ be the set of all nonnegative integers. We will use $n$, $m$ and $k$ to denote nonnegative integers. Unless otherwise stated, 'for all $n$ ' will mean 'for all $n \geq 0$.

Definition 1 (Roldán et al. [7]) A preorder (or a quasiorder) $\preccurlyeq$ on $X$ is a binary relation on $X$ that is reflexive (i.e., $x \preccurlyeq x$ for all $x \in X$ ) and transitive (if $x, y, z \in X$ verify $x \preccurlyeq y$ and $y \preccurlyeq z$, then $x \preccurlyeq z$ ). In such a case, we say that $(X, \preccurlyeq)$ is a preordered space (or a preordered set). If a preorder $\preccurlyeq$ is also antisymmetric ( $x \preccurlyeq y$ and $y \preccurlyeq x$ implies $x=y$ ), then $\preccurlyeq$ is called a partial order.

Throughout this manuscript, let $(X, d)$ be a metric space and let $\preccurlyeq$ be a preorder (or a partial order) on $X$. In the sequel, $T, g: X \rightarrow X$ and $F: X^{n} \rightarrow X$ will denote mappings.

Definition 2 A point $\left(x_{1}, x_{2}, \ldots, x_{n}\right) \in X^{n}$ is

- a coupled coincidence point of $F$ and $g$ if $n=2, F\left(x_{1}, x_{2}\right)=g x_{1}$ and $F\left(x_{2}, x_{1}\right)=g x_{2}$;

- a tripled coincidence point of $F$ and $g$ if $n=3, F\left(x_{1}, x_{2}, x_{3}\right)=g x_{1}, F\left(x_{2}, x_{1}, x_{2}\right)=g x_{2}$ and $F\left(x_{3}, x_{2}, x_{1}\right)=g x_{3}$;

- a quadrupled coincidence point of $F$ and $g$ if $n=4, F\left(x_{1}, x_{2}, x_{3}, x_{4}\right)=g x_{1}$, $F\left(x_{2}, x_{3}, x_{4}, x_{1}\right)=g x_{2}, F\left(x_{3}, x_{4}, x_{1}, x_{2}\right)=g x_{3}$ and $F\left(x_{4}, x_{1}, x_{2}, x_{3}\right)=g x_{4}$.

If $g$ is the identity mapping on $X$, then a point verifying the previous conditions is a coupled (respectively, tripled, quadrupled) fixed point of $F$ due to Gnana-Bhaskar and Lakshmikantham [1] (respectively, Berinde and Borcut [2], Karapınar [3]).

Definition 3 If $(X, \preccurlyeq)$ is a preordered space and $T, g: X \rightarrow X$ are two mappings, we will say that $T$ is a $(g, \preccurlyeq)$-nondecreasing mapping if $T x \preccurlyeq T y$ for all $x, y \in X$ such that $g x \preccurlyeq g y$. If $g$ is the identity mapping on $X, T$ is $\preccurlyeq$-nondecreasing.

In [8], the author called $g$-isotone to $(g, \preccurlyeq)$-nondecreasing mappings, especially in the case in which $X$ is a product space $X^{n}$.

Definition 4 A fixed point of a self-mapping $T: X \rightarrow X$ is a point $x \in X$ such that $T x=x$. A coincidence point of two mappings $T, g: X \rightarrow X$ is a point $x \in X$ such that $T x=g x$. A common fixed point of $T, g: X \rightarrow X$ is a point $x \in X$ such that $T x=g x=x$.

Definition 5 We will say that $T$ and $g$ are commuting if $g T x=T g x$ for all $x \in X$, and we will say that $F$ and $g$ are commuting if $g F\left(x_{1}, x_{2}, \ldots, x_{n}\right)=F\left(g x_{1}, g x_{2}, \ldots, g x_{n}\right)$ for all $x_{1}, \ldots, x_{n} \in X$.

Remark 6 If $T, g: X \rightarrow X$ are commuting and $x_{0} \in X$ is a coincidence point of $T$ and $g$, then $T x_{0}$ is also a coincidence point of $T$ and $g$.

The following notion was introduced in order to avoid the necessity of commutativity.

Definition 7 ([9-11]) Let $(X, d)$ be a metric space provided with a partial order $\preccurlyeq$. Two mappings $T, g: X \rightarrow X$ are said to be $O$-compatible if

$$
\lim _{m \rightarrow \infty} d\left(g \operatorname{Tx}, \operatorname{Tg} x_{m}\right)=0
$$


provided that $\left\{x_{m}\right\}$ is a sequence in $X$ such that $\left\{g x_{m}\right\}$ is $\preccurlyeq$-monotone and

$$
\lim _{m \rightarrow \infty} T x_{m}=\lim _{m \rightarrow \infty} g x_{m} \in X
$$

In 2003, Ran and Reuring proved the following version of the Banach theorem applicable to metric spaces endowed with a partial order.

Theorem 8 (Ran and Reurings [12]) Let $(X, \preccurlyeq)$ be an ordered set endowed with a metricd and $T: X \rightarrow X$ be a given mapping. Suppose that the following conditions hold:

(a) $(X, d)$ is complete.

(b) $T$ is $\preccurlyeq$-nondecreasing.

(c) $T$ is continuous.

(d) There exists $x_{0} \in X$ such that $x_{0} \preccurlyeq T x_{0}$.

(e) There exists a constant $k \in(0,1)$ such that $d(T x, T y) \leq k d(x, y)$ for all $x, y \in X$ with $x \succcurlyeq y$.

Then $T$ has a fixed point. Moreover, iffor all $(x, y) \in X^{2}$ there exists $z \in X$ such that $x \preccurlyeq z$ and $y \preccurlyeq z$, we obtain uniqueness of the fixed point.

Later, Nieto and Rodríguez-López [13] slightly modified the hypothesis of the previous result swapping condition (c) by the fact that $(X, d, \preccurlyeq)$ is nondecreasing-regular in the following sense.

Definition 9 Let $(X, \preccurlyeq)$ be an ordered set endowed with a metric $d$. We will say that $(X, d, \preccurlyeq)$ is nondecreasing-regular (respectively, nonincreasing-regular) if any $\preccurlyeq$ nondecreasing (respectively, $\preccurlyeq$-nonincreasing) sequence $\left\{x_{m}\right\} d$-convergent to $x \in X$, we have $x_{m} \preccurlyeq x$ (respectively, $\left.x_{m} \succcurlyeq x\right)$ for all $m$. $(X, d, \preccurlyeq)$ is regular if it is both nondecreasingregular and nonincreasing-regular.

Inspired by Boyd and Wong's theorem [14], Mukherjea [15] introduced the following kind of control functions:

$$
\Phi=\left\{\varphi:[0, \infty) \rightarrow[0, \infty): \varphi(t)<t \text { and } \lim _{r \rightarrow t^{+}} \varphi(r)<t \text { for each } t>0\right\},
$$

and he proved a version of the following result in which the space is not necessarily endowed with a partial order (but the contractivity condition holds over all pairs of points of the space).

Theorem 10 Let $(X, \preccurlyeq)$ be an ordered set endowed with a metric $d$ and $T: X \rightarrow X$ be a given mapping. Suppose that the following conditions hold:

(a) $(X, d)$ is complete.

(b) $T$ is $\preccurlyeq$-nondecreasing.

(c) Either $T$ is continuous or $(X, d, \preccurlyeq)$ is nondecreasing-regular.

(d) There exists $x_{0} \in X$ such that $x_{0} \preccurlyeq T x_{0}$.

(e) There exists $\varphi \in \Phi$ such that $d(T x, T y) \leq \varphi(d(x, y))$ for all $x, y \in X$ with $x \succcurlyeq y$.

Then $T$ has a fixed point. Moreover, iffor all $(x, y) \in X^{2}$ there exists $z \in X$ such that $x \preccurlyeq z$ and $y \preccurlyeq z$, we obtain uniqueness of the fixed point. 
An interesting version of the previous result in the coupled case for compatible mappings is the following one.

Theorem 11 (Choudhury and Kundu [9], Theorem 3.1) Let $(X, \preccurlyeq)$ be a partially ordered set and let there be a metric $d$ on $X$ such that $(X, d)$ is a complete metric space. Let $\varphi$ : $[0, \infty) \rightarrow[0, \infty)$ be such that $\varphi(t)<t$ and $\lim _{r \rightarrow t^{+}} \varphi(r)<t$ for all $t>0$. Let $F: X \times X \rightarrow X$ and $g: X \rightarrow X$ be two mappings such that $F$ has the mixed $g$-monotone property and satisfy

$$
d(F(x, y), F(u, v)) \leq \varphi\left(\frac{d(g x, g u)+d(g y, g v)}{2}\right)
$$

for all $x, y, u, v \in X$ with $g x \preccurlyeq g u$ and $g y \succcurlyeq g v$.

Let $F(X \times X) \subseteq g(X), g$ be continuous and monotone increasing and $F$ and $g$ be compatible mappings. Also suppose

(a) $F$ is continuous or

(b) $X$ has the following properties:

(i) if a nondecreasing sequence $\left\{x_{n}\right\} \rightarrow x$, then $x_{n} \preccurlyeq x$ for all $n \geq 0$;

(ii) if a nonincreasing sequence $\left\{y_{n}\right\} \rightarrow y$, then $y_{n} \succcurlyeq y$ for all $n \geq 0$.

If there exist $x_{0}, y_{0} \in X$ such that $g x_{0} \preccurlyeq F\left(x_{0}, y_{0}\right)$ and $g y_{0} \succcurlyeq F\left(y_{0}, x_{0}\right)$, then there exist $x, y \in X$ such that $g x=F(x, y)$ and $g y=F(y, x)$, that is, $F$ and $g$ have a coupled coincidence point in $X$.

A partial order $\preccurlyeq$ on $X$ can be extended to a partial order $\sqsubseteq$ on $X^{n}$ defining, for all $Y=\left(y_{1}, y_{2}, \ldots, y_{n}\right), V=\left(v_{1}, v_{2}, \ldots, v_{n}\right) \in X^{n}$,

$$
Y \sqsubseteq V \quad \text { if } \begin{cases}y_{i} \preccurlyeq v_{i}, & i=1,3,5, \ldots \\ y_{i} \succcurlyeq v_{i}, & i=2,4,6, \ldots\end{cases}
$$

Another interesting generalization of Theorem 10 was given by Wang in [8] using this extended partial order on $X^{n}$.

Theorem 12 (Wang [8], Theorem 3.4) Let $(X, \preccurlyeq)$ be a partially ordered set and suppose there is a metric $d$ on $X$ such that $(X, d)$ is a complete metric space. Let $G: X^{n} \rightarrow X^{n}$ and $T: X^{n} \rightarrow X^{n}$ be a G-isotone mapping for which there exists $\varphi \in \Phi$ such that for all $Y \in X^{n}$, $V \in X^{n}$ with $G(V) \sqsubseteq G(Y)$,

$$
\rho_{n}(T(Y), T(V)) \leq \varphi\left(\rho_{n}(G(Y), G(V))\right)
$$

where $\rho_{n}$ is defined, for all $Y=\left(y_{1}, y_{2}, \ldots, y_{n}\right), V=\left(v_{1}, v_{2}, \ldots, v_{n}\right) \in X^{n}$, by

$$
\rho_{n}(Y, V)=\frac{1}{n}\left[d\left(y_{1}, v_{1}\right)+d\left(y_{2}, v_{2}\right)+\cdots+d\left(y_{n}, v_{n}\right)\right] .
$$

Suppose $T\left(X^{n}\right) \subseteq G\left(X^{n}\right)$ and also suppose either

(a) $T$ is continuous, $G$ is continuous and commutes with $T$, or

(b) $(X, d, \preccurlyeq)$ is regular and $G\left(X^{n}\right)$ is closed.

If there exists $Y_{0} \in X^{n}$ such that $G\left(Y_{0}\right)$ and $T\left(Y_{0}\right)$ are $\sqsubseteq$-comparable, then $T$ and $G$ have a coincidence point. 
Some other generalizations of the previous result can be found in Romaguera [16] (to partial metric spaces, but not necessarily provided with a partial order).

In order to guarantee the existence and uniqueness of a solution of periodic boundary value problems, Gnana Bhaskar and Lakshmikantham (and, subsequently, Lakshmikantham and Ćirić; see [17]) proved, in 2006, existence and uniqueness of a coupled fixed point (a notion introduced by Guo and Laksmikantham [18]) in the setting of partially ordered metric spaces by introducing the notion of mixed monotone property.

In order to ensure the existence of coupled fixed points, Gnana Bhaskar and Lakshmikantham introduced the following condition.

Definition 13 (Gnana Bhaskar and Lakshmikantham [1]) Let $(X, \preccurlyeq)$ be a partially ordered set and $F: X \times X \rightarrow X$. We say that $F$ has the mixed monotone property if $F(x, y)$ is monotone nondecreasing in $x$ and is monotone nonincreasing in $y$, that is, for any $x, y \in X$,

$$
\begin{array}{ll}
x_{1}, x_{2} \in X, \quad x_{1} \preccurlyeq x_{2} \quad \Rightarrow \quad F\left(x_{1}, y\right) \preccurlyeq F\left(x_{2}, y\right), \\
y_{1}, y_{2} \in X, \quad y_{1} \preccurlyeq y_{2} \quad \Rightarrow \quad F\left(x, y_{1}\right) \succcurlyeq F\left(x, y_{2}\right) .
\end{array}
$$

Many result were proved to ensure the existence of coupled fixed point. One of the common properties of all these results is the fact that the mapping $F: X \times X \rightarrow X$ must verify the mixed monotone property. Searching for a generalization of this kind of theorems, Samet and Vetro [19] succeeded in proving some results in which the mapping $F$ did not necessarily have the mixed monotone property.

Definition 14 (Samet and Vetro [19]) Let $(X, d)$ be a metric space and $F: X \times X \rightarrow X$ be a given mapping. Let $M$ be a nonempty subset of $X^{4}$. We say that $M$ is an $F$-invariant subset of $X^{4}$ if, for all $x, y, z, w \in X$,

(i) $(x, y, z, w) \in M \Longleftrightarrow(w, z, y, x) \in M$;

(ii) $(x, y, z, w) \in M \Longrightarrow(F(x, y), F(y, x), F(z, w), F(w, z)) \in M$.

The following theorem is the main result in [19].

Theorem 15 (Samet and Vetro [19]) Let $(X, d)$ be a complete metric space, $F: X \times X \rightarrow X$ be a continuous mapping and $M$ be a nonempty subset of $X^{4}$. We assume that

(i) $M$ is F-invariant;

(ii) there exists $\left(x_{0}, y_{0}\right) \in X^{2}$ such that $\left(F\left(x_{0}, y_{0}\right), F\left(y_{0}, x_{0}\right), x_{0}, y_{0}\right) \in M$;

(iii) for all $(x, y, u, v) \in M$, we have

$$
\begin{aligned}
d(F(x, y), F(u, v)) \leq & \frac{\alpha}{2}[d(x, F(x, y))+d(y, F(y, x))] \\
& +\frac{\beta}{2}[d(u, F(u, v))+d(v, F(v, u))] \\
& +\frac{\theta}{2}[d(x, F(u, v))+d(y, F(v, u))] \\
& +\frac{\gamma}{2}[d(u, F(x, y))+d(v, F(y, x))]+\frac{\delta}{2}[d(x, u)+d(y, v)],
\end{aligned}
$$

where $\alpha, \beta, \theta, \gamma, \delta$ are nonnegative constants such that $\alpha+\beta+\theta+\gamma+\delta<1$. 
Then $F$ has a coupled fixed point, i.e., there exists $(x, y) \in X \times X$ such that $F(x, y)=x$ and $F(y, x)=y$.

Later, Sintunaravat et al. [20] introduced the notion of transitive property so as to extend the Lakshmikantham and Ćirić's theorem (see [17]).

Definition 16 (Sintunaravat et al. [20]) Let $(X, d)$ be a metric space and $M$ be a subset of $X^{4}$. We say that $M$ satisfies the transitive property if, for all $x, y, z, w, a, b \in X$,

$$
(x, y, z, w) \in M \quad \text { and } \quad(z, w, a, b) \in M \quad \Longrightarrow \quad(x, y, a, b) \in M
$$

Then they proved the following result.

Theorem 17 (Sintunaravat et al. [20]) Let $(X, d)$ be a complete metric space and $M$ be a nonempty subset of $X^{4}$. Assume that there is a function $\varphi:[0, \infty) \rightarrow[0, \infty)$ with $0=$ $\varphi(0)<\varphi(t)<t$ and $\lim _{r \rightarrow t^{+}} \varphi(r)<t$ for each $t>0$, and also suppose that $F: X \times X \rightarrow X$ is a mapping such that

$$
d(F(x, y), F(u, v)) \leq \varphi\left(\frac{d(x, u)+d(y, v)}{2}\right)
$$

for all $(x, y, u, v) \in M$. Suppose that either

(a) $F$ is continuous or

(b) for any two sequences $\left\{x_{m}\right\},\left\{y_{m}\right\}$ with $\left(x_{m+1}, y_{m+1}, x_{m}, y_{m}\right) \in M$,

$$
\left\{x_{m}\right\} \rightarrow x, \quad\left\{y_{m}\right\} \rightarrow y,
$$

for all $m \geq 1$, then $\left(x, y, x_{m}, y_{m}\right) \in M$ for all $m \geq 1$.

If there exists $\left(x_{0}, y_{0}\right) \in X \times X$ such that $\left(F\left(x_{0}, y_{0}\right), F\left(y_{0}, x_{0}\right), x_{0}, y_{0}\right) \in M$ and $M$ is an $F$ invariant set which satisfies the transitive property, then there exist $x, y \in X$ such that $x=$ $F(x, y)$ and $y=F(y, x)$, that is, $F$ has a coupled fixed point.

In recent times, it has been proved that many coupled, tripled, and quadrupled results can be reduced to the unidimensional case, that is, to Theorems 8 and 10 (see, for instance, Samet et al. [21], Agarwal et al. [22] and Roldán et al. [6], Ding et al. [23], Karapınar [24] and Karapınar and Roldán [25]). Furthermore, in some cases, it is not necessary to consider a partial order, but a preorder (see Roldán et al. [7]).

Recently, in 2013, Batra and Vashistha [26] introduced the concept of $(F, g)$-invariant set which is a generalization of an $F$-invariant set introduced by Samet and Vetro [19] and proved the existence of coupled common fixed point theorems for nonlinear contractions under $c$-distance in cone metric spaces having an $(F, g)$-invariant subset.

More recently, Charoensawan [27], based on Batra and Vashistha's results, introduced the tripled case as follows.

Definition 18 (Charoensawan [27]) Let $(X, \preccurlyeq)$ be a metric space and $F: X^{3} \rightarrow X$ be a given mapping. Let $M$ be a nonempty subset of $X^{6}$. We say that $M$ is an $F$-invariant subset of $X^{6}$ if, and only if, for all $x, y, z, u, v, w \in X$,

$$
\begin{aligned}
& (x, y, z, u, v, w) \in M \\
& \quad \Longrightarrow \quad(F(x, y, z), F(y, x, y), F(z, y, x), F(u, v, w), F(v, u, v), F(w, v, u)) \in M .
\end{aligned}
$$


The following concept is an extension of Definition 16.

Definition 19 (Charoensawan [27]) Let $(X, \preccurlyeq)$ be a metric space and $M$ be a subset of $X^{6}$. We say that $M$ satisfies the transitive property if, and only if, for all $x, y, z, u, v, w, a, b, c \in X$,

$$
(x, y, z, u, v, w) \in M \quad \text { and } \quad(u, v, w, a, b, c) \in M \quad \Longrightarrow \quad(x, y, z, a, b, c) \in M .
$$

Definition 20 (Charoensawan [27]) Let $(X, \preccurlyeq)$ be a metric space and $F: X^{3} \rightarrow X, g: X \rightarrow$ $X$ be given mappings. Let $M$ be a nonempty subset of $X^{6}$. We say that $M$ is an $(F, g)$ invariant subset of $X^{6}$ if, and only if, for all $x, y, z, u, v, w \in X$,

$$
\begin{aligned}
& (g x, g y, g z, g u, g v, g w) \in M \\
& \quad \Longrightarrow \quad(F(x, y, z), F(y, x, y), F(z, y, x), F(u, v, w), F(v, u, v), F(w, v, u)) \in M .
\end{aligned}
$$

Notice that in the previous definitions, it is not necessary to consider neither a metric nor a partial order on $X$. Then this author proved the following result.

Theorem 21 (Charoensawan [27], Theorem 3.7) Let $(X, \preccurlyeq)$ be a complete metric space and $M$ be a nonempty subset of $X^{6}$. Assume that there is a function $\varphi:[0,+\infty) \rightarrow[0,+\infty)$ with $0=\varphi(0)<\varphi(t)<$ tand $\lim _{r \rightarrow t^{+}} \varphi(r)<t$ for each $t>0$, and also suppose that $F: X^{3} \rightarrow X$ and $g: X \rightarrow X$ are two continuous functions such that

$$
\begin{aligned}
& d(F(x, y, z), F(u, v, w))+d(F(y, x, y), F(v, u, v))+d(F(z, y, x), F(w, v, u)) \\
& \quad \leq 3 \varphi\left(\frac{d(g x, g u)+d(g y, g v)+d(g z, g w)}{3}\right)
\end{aligned}
$$

for all $x, y, z, u, v, w \in X$ with $(g x, g y, g z, g u, g v, g w) \in M$ or $(g u, g v, g w, g x, g y, g z) \in M$. Suppose that $F\left(X^{3}\right) \subseteq g X, g$ commutes with $F$.

If there exists $\left(x_{0}, y_{0}, z_{0}\right) \in X^{3}$ such that

$$
\left(F\left(x_{0}, y_{0}, z_{0}\right), F\left(y_{0}, x_{0}, y_{0}\right), F\left(z_{0}, y_{0}, x_{0}\right), g x_{0}, g y_{0}, g z_{0}\right) \in M
$$

and $M$ is an $(F, g)$-invariant set which satisfies the transitive property, then there exist $x, y, z \in X$ such that

$$
g x=F(x, y, z), \quad g y=F(y, x, y) \quad \text { and } \quad g z=F(z, y, x) .
$$

Not knowing them and independently from Charoensawan's results, Kutbi et al. [28] used a bidimensional extension of $F$-invariant subset as follows.

Definition 22 (Kutbi et al. [28]) We say that $M$ is an F-closed subset of $X^{4}$ if, for all $x, y, u, v \in X$,

$$
(x, y, u, v) \in M \quad \Longrightarrow \quad(F(x, y), F(y, x), F(u, v), F(v, u)) \in M .
$$

Then they succeeded in proving some results using this property rather than the mixed monotone property and the concept of $F$-invariant set. For instance, the following one. 
Theorem 23 (Kutbi et al. [28]) Let $(X, d)$ be a complete metric space, let $F: X \times X \rightarrow X$ be a continuous mapping and let $M$ be a subset of $X^{4}$. Assume the following.

(i) $M$ is F-closed;

(ii) there exists $\left(x_{0}, y_{0}\right) \in X^{2}$ such that $\left(F\left(x_{0}, y_{0}\right), F\left(y_{0}, x_{0}\right), x_{0}, y_{0}\right) \in M$;

(iii) there exists $k \in[0,1)$ such that for all $(x, y, u, v) \in M$, we have

$$
d(F(x, y), F(u, v))+d(F(y, x), F(v, u)) \leq k(d(x, u)+d(y, v)) .
$$

Then $F$ has a coupled fixed point.

The following two lemmas can be found in the literature, but we recall them here for the sake of completeness.

Lemma 24 Let $\varphi \in \Phi$ be a mapping and let $\left\{a_{m}\right\} \subset \mathbb{R}_{0}^{+}$be a sequence.

1. If $a_{m+1} \leq \varphi\left(a_{m}\right)$ and $a_{m} \neq 0$ for all $m$, then $\left\{a_{m}\right\} \rightarrow 0$.

2. If $\varphi(0)=0$ and $\left\{b_{m}\right\} \subset \mathbb{R}_{0}^{+}$is a sequence verifying $a_{m} \leq \varphi\left(b_{m}\right)$ for all $m$ and $\left\{b_{m}\right\} \rightarrow 0$, then $\left\{a_{m}\right\} \rightarrow 0$.

Lemma 25 If $\left\{x_{m}\right\}_{m \in \mathbb{N}}$ is a sequence on a metric space $(X, d)$ that is not Cauchy, then there exist $\varepsilon_{0}>0$ and two subsequences $\left\{x_{m(k)}\right\}_{k \in \mathbb{N}}$ and $\left\{x_{n(k)}\right\}_{k \in \mathbb{N}}$ such that, for all $k \in \mathbb{N}$ :

$$
k \leq m(k)<n(k), \quad d\left(x_{m(k)}, x_{n(k)-1}\right) \leq \varepsilon_{0}<d\left(x_{m(k)}, x_{n(k)}\right) \quad \text { for all } k .
$$

Furthermore, if $\left\{d\left(x_{m}, x_{m+1}\right)\right\} \rightarrow 0$, then

$$
\lim _{k \rightarrow \infty} d\left(x_{m(k)}, x_{n(k)}\right)=\lim _{k \rightarrow \infty} d\left(x_{m(k)+1}, x_{n(k)+1}\right)=\varepsilon_{0} .
$$

In this paper we observe that if $M \subseteq X^{4}$ is $F$-invariant and has the transitive property, we could induce a preorder on $X^{2}$ such that Theorem 17 can be seen as an easy consequence of Theorem 10. Actually, we will present an unidimensional result that can be particularized, following well-known techniques, to the multidimensional case.

\section{Main results}

Before showing our main results, some remarks must be done. Firstly, consider the family

$$
\Phi^{\prime}=\left\{\varphi:[0, \infty) \rightarrow[0, \infty): 0=\varphi(0)<\varphi(t)<t \text { and } \lim _{r \rightarrow t^{+}} \varphi(r)<t \text { for each } t>0\right\} .
$$

This family of control functions was employed by Sintunaravat et al. in Theorem 17 and by Charoensawan in Theorem 21. However, notice that it is not as general as Wang's family $\Phi$ because the value $\varphi(0)$ is not necessarily determined if $\varphi \in \Phi$. In this sense, $\Phi^{\prime} \subset \Phi$.

Secondly, notice that Charoensawan's notion of $F$-invariant set is similar to Kutbi et al.'s notion of F-closed set, but it is different from Samet and Vetro's original concept because property (i) in Definition 14 is not imposed. Then, coherently with Definition 22, we prefer calling these subsets employing the term F-closed.

Definition 26 Let $T, g: X \rightarrow X$ be two mappings and let $M \subseteq X^{2}$ be a subset. We will say that $M$ is 
- $(T, g)$-closed if $(T x, T y) \in M$ for all $x, y \in X$ such that $(g x, g y) \in M$;

- $(T, g)$-compatible if $T x=T y$ for all $x, y \in X$ such that $g x=g y$.

Definition 27 We will say that a subset $M \subseteq X^{2}$ is transitive if $(x, y),(y, z) \in M$ implies that $(x, z) \in M$.

Definition 28 Let $(X, d)$ be a metric space and let $M \subseteq X^{2}$ be a subset. We will say that $(X, d, M)$ is regular if for all sequence $\left\{x_{m}\right\} \subseteq X$ such that $\left\{x_{m}\right\} \rightarrow x$ and $\left(x_{m}, x_{m+1}\right) \in M$ for all $m$, we have $\left(x_{m}, x\right) \in M$ for all $m$.

We introduce a notion of continuity weaker than the usual concept.

Definition 29 Let $(X, d)$ be a metric space, let $M \subseteq X^{2}$ be a subset and let $x \in X$. A mapping $T: X \rightarrow X$ is said to be M-continuous at $x$ if for all sequence $\left\{x_{m}\right\} \subseteq X$ such that $\left\{x_{m}\right\} \rightarrow x$ and $\left(x_{m}, x_{m+1}\right) \in M$ for all $m$, we have $\left\{T x_{m}\right\} \rightarrow T x . T$ is $M$-continuous if it is $M$-continuous at each $x \in X$.

Remark 30 Every continuous mapping is also $M$-continuous, whatever $M$.

In order to avoid the commutativity condition of the mappings $T$ and $g$, and inspired by Definition 7, we present the following notion of $(O, M)$-compatibility.

Definition 31 Let $(X, d)$ be a metric space and let $M \subseteq X^{2}$ be a subset. Two mappings $T, g: X \rightarrow X$ are said to be $(O, M)$-compatible if

$$
\lim _{m \rightarrow \infty} d\left(g T x_{m}, \operatorname{Tg} x_{m}\right)=0
$$

provided that $\left\{x_{m}\right\}$ is a sequence in $X$ such that $\left(g x_{m}, g x_{m+1}\right) \in M$ for all $m \geq 0$ and

$$
\lim _{m \rightarrow \infty} T x_{m}=\lim _{m \rightarrow \infty} g x_{m} \in X
$$

Remark 32 If $T$ and $g$ are commuting, then they are also (O,M)-compatible, whatever $M$.

The main result of this paper is the following one.

Theorem 33 Let $(X, d)$ be a complete metric space, let $T, g: X \rightarrow X$ be two mappings such that $T X \subseteq g X$ and let $M \subseteq X^{2}$ be a $(T, g)$-compatible, $(T, g)$-closed, transitive subset. Assume that there exists $\varphi \in \Phi$ such that

$$
d(T x, T y) \leq \varphi(d(g x, g y)) \quad \text { for all } x, y \in X \text { such that }(g x, g y) \in M
$$

Also assume that, at least, one of the following conditions holds.

(a) $T$ and $g$ are $M$-continuous and $(O, M)$-compatible;

(b) $T$ and $g$ are continuous and commuting;

(c) $(X, d, M)$ is regular and $g X$ is closed.

If there exists a point $x_{0} \in X$ such that $\left(g x_{0}, T x_{0}\right) \in M$, then $T$ and $g$ have, at least, $a$ coincidence point. 
Proof Starting from $x_{0}$ such that $\left(g x_{0}, T x_{0}\right) \in M$, we have $T x_{0} \in T X \subseteq g X$, so there exists $x_{1} \in X$ such that $g x_{1}=T x_{0}$. Therefore $\left(g x_{0}, g x_{1}\right)=\left(g x_{0}, T x_{0}\right) \in M$. As $M$ if $(T, g)$-closed, $\left(T x_{0}, T x_{1}\right) \in M$. Again, since $T x_{1} \in T X \subseteq g X$, there exists $x_{2} \in X$ such that $g x_{2}=T x_{1}$. Therefore $\left(g x_{1}, g x_{2}\right)=\left(T x_{0}, T x_{1}\right) \in M$. As $M$ if $(T, g)$-closed, $\left(T x_{1}, T x_{2}\right) \in M$. Repeating this process, there exists a sequence $\left\{x_{m}\right\} \subseteq X$ such that

$$
g x_{m+1}=T x_{m} \quad \text { and } \quad\left(g x_{m}, g x_{m+1}\right) \in M \quad \text { for all } m \geq 0 .
$$

If there exists $m_{0} \in \mathbb{N}$ such that $g x_{m_{0}}=g x_{m_{0}+1}$, then $g x_{m_{0}}=g x_{m_{0}+1}=T x_{m_{0}}$, so $x_{m_{0}}$ is a coincidence point of $T$ and $g$. In this case, the proof is finished. On the contrary, assume that

$$
g x_{m} \neq g x_{m+1}, \quad \text { that is, } \quad d\left(g x_{m}, g x_{m+1}\right)>0 \quad \text { for all } m \text {. }
$$

As $\left(g x_{m}, g x_{m+1}\right) \in M$ for all $m$, the contractivity condition (5) implies that

$$
d\left(g x_{m+1}, g x_{m+2}\right)=d\left(T x_{m}, T x_{m+1}\right) \leq \varphi\left(d\left(g x_{m}, g x_{m+1}\right)\right) \quad \text { for all } m .
$$

Applying (7) and item 1 of Lemma 24 to $\left\{a_{m}=d\left(g x_{m}, g x_{m+1}\right)\right\}$, we deduce that

$$
\left\{d\left(g x_{m}, g x_{m+1}\right)\right\} \rightarrow 0
$$

Next, we show that $\left\{g x_{m}\right\}$ is a Cauchy sequence reasoning by contradiction. Suppose that $\left\{g x_{m}\right\}$ is not a Cauchy sequence. Taking into account (8), a well-known reasoning guarantees that there exist $\varepsilon_{0}>0$ and two partial subsequences $\left\{g x_{m(k)}\right\}$ and $\left\{g x_{n(k)}\right\}$ such that

$$
\begin{aligned}
& k \leq m(k)<n(k), \quad d\left(g x_{m(k)}, g x_{n(k)-1}\right) \leq \varepsilon_{0}<d\left(g x_{m(k)}, g x_{n(k)}\right) \quad \text { for all } k, \\
& \lim _{k \rightarrow \infty} d\left(g x_{m(k)}, g x_{n(k)}\right)=\lim _{k \rightarrow \infty} d\left(g x_{m(k)+1}, g x_{n(k)+1}\right)=\varepsilon_{0} .
\end{aligned}
$$

Since $M$ is transitive, it follows from (6) that $\left(g x_{m(k)}, g x_{n(k)}\right) \in M$ for all $k$. Let us apply the contractivity condition (5) to $x=g x_{m(k)}$ and $y=g x_{n(k)}$, and we get, for all $k$,

$$
d\left(g x_{m(k)+1}, g x_{n(k)+1}\right)=d\left(T x_{m(k)}, T x_{n(k)}\right) \leq \varphi\left(d\left(g x_{m(k)}, g x_{n(k)}\right)\right) .
$$

By (9) and (10), $\left\{d\left(g x_{m(k)}, g x_{n(k)}\right)\right\}$ is a sequence of real numbers, stricly greater than $\varepsilon_{0}$, that converges to $\varepsilon_{0}$. In particular, since $\varphi \in \Phi$,

$$
\lim _{k \rightarrow \infty} \varphi\left(d\left(g x_{m(k)}, g x_{n(k)}\right)\right)=\lim _{s \rightarrow \varepsilon_{0}^{+}} \varphi(s)<\varepsilon_{0}
$$

Letting $k \rightarrow \infty$ in (11) and using (10) and (12), we deduce that

$$
\varepsilon_{0}=\lim _{k \rightarrow \infty} d\left(g x_{m(k)+1}, g x_{n(k)+1}\right) \leq \lim _{k \rightarrow \infty} \varphi\left(d\left(g x_{m(k)}, g x_{n(k)}\right)\right)<\varepsilon_{0},
$$

which is impossible. This contradiction proves that $\left\{g x_{m}\right\}$ is a Cauchy sequence. 
As $(X, d)$ is complete, there is $x \in X$ such that $\left\{g x_{m}\right\} \rightarrow x$. Next we distinguish between hypotheses (a), (b), and (c).

Case (a). Assume that $T$ and $g$ are $M$-continuous and $(O, M)$-compatible. Since $\left\{g x_{m}\right\} \rightarrow$ $x$ and $\left(g x_{m}, g x_{m+1}\right) \in M$ for all $m \geq 0$, we have $\left\{g g x_{m}\right\} \rightarrow g x$ and $\left\{T g x_{m}\right\} \rightarrow T x$. Furthermore, as $T$ and $g$ are $(O, M)$-compatible, we have $\lim _{m \rightarrow \infty} d\left(g T x_{m}, \operatorname{Tg} x_{m}\right)=0$. Therefore,

$$
d(g x, T x)=\lim _{m \rightarrow \infty} d\left(g T x_{m}, \operatorname{Tg} x_{m}\right)=0,
$$

which means that $x$ is a coincidence point of $T$ and $g$.

Case (b). By Remarks 30 and 32, if $T$ and $g$ are continuous and commuting, then they are also $M$-continuous and $(O, M)$-compatible, so item (a) is applicable.

Case (c). Now, suppose that $(X, d, M)$ is regular and $g X$ is closed. As $\left\{g x_{m}\right\} \subseteq g X$ and $g X$ is closed, then $x \in g X$, that is, there is $z \in X$ such that $g z=x$. Taking into account that $(X, d, M)$ is regular, then $\left(g x_{m}, g z\right)=\left(g x_{m}, x\right) \in M$ for all $m$. Applying the contractivity condition (5), we have

$$
d\left(g x_{m+1}, T z\right)=d\left(T x_{m}, T z\right) \leq \varphi\left(d\left(g x_{m}, g z\right)\right)=\varphi\left(d\left(g x_{m}, x\right)\right) \quad \text { for all } m
$$

Now we distinguish whether $\varphi(0)=0$ or not. If $\varphi(0)=0$, item 2 of Lemma 24 (applied to $a_{m}=d\left(g x_{m+1}, T z\right)$ and $b_{m}=d\left(g x_{m}, x\right)$ for all $\left.m\right)$ ensures us that $\left\{d\left(g x_{m+1}, T z\right)\right\} \rightarrow 0$, that is, $\left\{g x_{m+1}\right\}$ converges to $T z$. In this case, $g z=x=\lim _{m \rightarrow \infty} g x_{m}=T z$, so $z$ is a coincidence point between $T$ and $g$. Next, suppose that $\varphi(0) \neq 0$ and we are going to show that

$$
d\left(g x_{m+1}, T z\right) \leq d\left(g x_{m}, x\right) \quad \text { for all } m \geq 1 .
$$

- If $d\left(g x_{m}, x\right) \neq 0$, then $d\left(g x_{m+1}, T z\right) \leq \varphi\left(d\left(g x_{m}, x\right)\right)<d\left(g x_{m}, x\right)$ since $\varphi \in \Phi$.

- Suppose that there is some $m_{0} \in \mathbb{N}$ such that $d\left(g x_{m_{0}}, x\right)=0$. Then $g x_{m_{0}}=x=g z$ and $\left(g x_{m_{0}}, g z\right) \in M$. Taking into account that $M$ is $(T, g)$-compatible, we deduce that $T x_{m_{0}}=T z$. Therefore $g x_{m_{0}+1}=T x_{m_{0}}=T z$ and (13) also holds for $m_{0}$.

In any case, (13) holds for all $m \geq 1$. Therefore $\left\{g x_{m+1}\right\} \rightarrow T z$ and, by the unicity of the limit, $T z=g z$.

If one takes $\varphi_{k} \in \Phi$ in Theorem 33, where $k \in[0,1)$ and $\varphi_{k}(t)=k t$ for all $t \geq 0$, and remove the transitivity condition, we get the following statement.

Corollary 34 Let $(X, d)$ be a complete metric space, let $T, g: X \rightarrow X$ be two mappings such that $T X \subseteq g X$ and let $M \subseteq X^{2}$ be a $(T, g)$-compatible, $(T, g)$-closed set. Assume that there exists $k \in[0,1)$ such that

$$
d(T x, T y) \leq k d(g x, g y) \quad \text { for all } x, y \in X \text { such that }(g x, g y) \in M .
$$

Also assume that, at least, one of the following conditions holds.

(a) $T$ and $g$ are $M$-continuous and $(O, M)$-compatible;

(b) $T$ and $g$ are continuous and commuting;

(c) $(X, d, M)$ is regular and $g X$ is closed.

If there exists a point $x_{0} \in X$ such that $\left(g x_{0}, T x_{0}\right) \in M$, then $T$ and $g$ have, at least, $a$ coincidence point. 
Proof We use the same structure as in the proof of Theorem 33. By following its lines, we derive

$$
d\left(g x_{m+1}, g x_{m+2}\right)=d\left(T x_{m}, T x_{m+1}\right) \leq k d\left(g x_{m}, g x_{m+1}\right) \text { for all } m \text {, }
$$

and, therefore,

$$
d\left(g x_{m}, g x_{m+1}\right) \leq k^{m} d\left(g x_{0}, g x_{1}\right) \quad \text { for all } m \text {. }
$$

By standard techniques, one can easily deduce that $\left\{g x_{m}\right\}$ is a Cauchy sequence (notice that it is not necessary the transitivity condition because we do not need to apply the contractivity condition to $\left.d\left(g x_{m(k)}, g x_{n(k)}\right)\right)$. Since $(X, d)$ is complete, there is $x \in X$ such that $\left\{g x_{m}\right\} \rightarrow x$. The rest is the same as in the proof of Theorem 33 .

We particularize the previous theorem, obtaining the following version of Theorem 12, in which a partial order is not necessary.

Corollary 35 Let $(X, d)$ be a complete metric space and let $\preccurlyeq$ be a transitive relation on $X$. Let $T, g: X \rightarrow X$ be two mappings such that $T X \subseteq g X$ and $T$ is $(g, \preccurlyeq)$-nondecreasing. Suppose that there exists $\varphi \in \Phi$ such that

$$
d(T x, T y) \leq \varphi(d(g x, g y)) \quad \text { for all } x, y \in X \text { such that } g x \preccurlyeq g y .
$$

Also suppose that

$$
\varphi(0)=0 \quad \text { or } \preccurlyeq \text { is antisymmetric. }
$$

\section{Assume that either}

(a) $T$ and $g$ are continuous and commuting, or

(b) $(X, d, \preccurlyeq)$ is regular and $g X$ is closed.

If there exists a point $x_{0} \in X$ such that $g x_{0} \preccurlyeq T x_{0}$, then $T$ and $g$ have, at least, a coincidence point.

Proof Consider the subset $M=\left\{(x, y) \in X^{2}: x \preccurlyeq y\right\}$. Then the following properties hold.

- $M$ is nonempty because $\left(g x_{0}, T x_{0}\right) \in M$.

- $M$ is transitive because $\preccurlyeq$ is so.

- If $x, y \in X$ are such that $(g x, g y) \in M$, then $g x \preccurlyeq g y$. Since $T$ is $(g, \preccurlyeq)$-nondecreasing, we have $T x \preccurlyeq T y$, that is, $(T x, T y) \in M$. Therefore, $M$ is $(T, g)$-closed.

- Let $x, y \in X$ be such that $(g x, g y) \in M$ and $g x=g y$. If $\varphi(0)=0$, then $d(T x, T y) \leq \varphi(0)=0$, so $T x=T y$. On the contrary, if $\preccurlyeq$ is antisymmetric, since $T$ is $(g, \preccurlyeq)$-nondecreasing, we have

$$
g x=g y, \quad(g x, g y) \in M \quad \Rightarrow \quad\left\{\begin{array}{lll}
g x \preccurlyeq g y & \Rightarrow & T x \preccurlyeq T y \\
g y \preccurlyeq g x & \Rightarrow & T y \preccurlyeq T x
\end{array}\right\} \Rightarrow T x=T y .
$$

In any case, $M$ is $(T, g)$-compatible.

- It is clear that $(X, d, M)$ is regular when $(X, d, \preccurlyeq)$ is regular. 
Then $M$ verifies all conditions of Theorem 33 and it guarantees that $T$ and $g$ have, at least, a coincidence point.

In the previous result, the condition $\varphi(0)=0$ does not mean that necessarily $\varphi \in \Phi^{\prime}$. For instance, if we define $\varphi_{0}(t)=0$ for all $t \geq 0$, then we have $\varphi_{0} \in \Phi \backslash \Phi^{\prime}$ and $\varphi_{0}(0)=0$.

Corollary 36 Theorem 12 immediately follows from Theorem 33.

Proof The result follows from Corollary 35 when we observe that if $(X, d, \preccurlyeq)$ is regular, then $\left(X^{n}, \rho_{n}, \sqsubseteq\right.$ ) is also regular (where $\rho_{n}$ is defined in Theorem 12 and $\sqsubseteq$ is given by $(1)$ ).

As we have pointed out in Preliminaries, recently, some authors have showed that many coupled, tripled, quadrupled and multidimensional fixed point results can be reduced to the unidimensional case. Next we show how coupled and tripled coincidence point results (for instance, Theorems 17 and 21) can be directly deduced from the 'unidimensional' Theorem 33 .

We introduce the following notation. Let $(X, \preccurlyeq)$ be a partially ordered space and let $n \in$ $\{2,3\}$. Given two mappings $g: X \rightarrow X$ and $F: X^{n} \rightarrow X$, define

$$
\begin{array}{ll}
T_{F}^{2}: X^{2} \rightarrow X^{2}, & T_{F}^{2}(x, y)=(F(x, y), F(y, x)) \\
G^{2}: X^{2} \rightarrow X^{2}, & G^{2}(x, y)=(g x, g y) ; \\
T_{F}^{3}: X^{3} \rightarrow X^{3}, & T_{F}^{3}(x, y, z)=(F(x, y, z), F(y, x, y), F(z, y, x)) \\
G^{3}: X^{3} \rightarrow X^{3}, & G^{3}(x, y, z)=(g x, g y, g z) .
\end{array}
$$

Clearly, a coincidence point between $F$ and $g$ is nothing but a coincidence point between $T_{F}^{n}$ and $G^{n}$.

A nonempty subset $M$ of $X^{2 n}$ is $(F, g)$-closed if $M$ is $\left(T_{F}^{n}, G^{n}\right)$-closed, that is,

- $n=2:(g x, g y, g u, g v) \in M \Longrightarrow(F(x, y), F(y, x), F(u, v), F(v, u)) \in M$;

- $n=3:(g x, g y, g z, g u, g v, g w) \in M \Longrightarrow(F(x, y, z), F(y, x, y), F(z, y, x), F(u, v, w), F(v, u, v)$, $F(w, v, u)) \in M$.

Corollary 37 Theorem 17 immediately follows from Theorem 33.

Proof It is only necessary to consider $g$ as the identity mapping on $X$ and the metric $D^{2}$ on $X^{2}$ given by

$$
D^{2}((x, y),(u, v))=\frac{d(x, u)+d(y, v)}{2} \quad \text { for all }(x, y),(u, v) \in X^{2} .
$$

Define

- $n=2:(x, y) \sqsubseteq_{M^{2}}(u, v) \Longleftrightarrow\left[(x, y)=(u, v)\right.$ or $\left.(u, v, x, y) \in M^{2}\right]$;

- $n=3:(x, y, z) \sqsubseteq_{M^{3}}(u, v, w) \Longleftrightarrow\left[(x, y, z)=(u, v, w)\right.$ or $\left.(u, v, w, x, y, z) \in M^{3}\right]$.

Definition 38 We will say that $M^{n} \subseteq X^{2 n}$ is $(F, g)$-compatible if $M^{n}$ is $\left(T_{F}^{n}, G^{n}\right)$-compatible (that is, $T_{F}^{n} A=T_{F}^{n} B$ for all $A, B \in M^{n}$ such that $G^{n} A=G^{n} B$ ). 
Lemma 39 Let $n \in\{2,3\}$, let $g: X \rightarrow X$ and $F: X^{n} \rightarrow X$ be two mappings and let $M^{n}$ be a nonempty subset of $X^{2 n}$. Then the following properties hold.

1. $\sqsubseteq M^{n}$ is reflexive whatever $M^{n}$.

2. $M^{n}$ satisfies the transitive property if, and only if, $\square_{M^{n}}$ is a preorder (reflexive and transitive) on $X^{2 n}$.

Next, assume that $M^{n}$ is $(F, g)$-compatible.

3. $M^{n}$ is $(F, g)$-closed if, and only if, the mapping $T_{F}^{n}$ is $\left(G^{n}\right.$, $\left.\sqsubseteq_{M^{n}}\right)$-nondecreasing.

4. If $M^{n}$ is F-invariant, then the mapping $T_{F}^{n}$ is $\sqsubseteq_{M^{n} \text {-nondecreasing. }}$

Proof We include the proof assuming that $n=2$ because the case $n=3$ is exactly the same.

(1) It is obvious. (2) Suppose that $M^{2}$ satisfies the transitive property and let $(x, y) \sqsubseteq_{M^{2}}$ $(u, v)$ and $(u, v) \sqsubseteq_{M^{2}}(a, b)$. If $(x, y)=(u, v)$ or $(u, v)=(a, b)$, then it is apparent that $(x, y) \sqsubseteq_{M^{2}}$ $(a, b)$. In other case, $(u, v, x, y) \in M^{2}$ and $(a, b, u, v) \in M^{2}$. Since $M^{2}$ satisfies the transitive property, then $(a, b, x, y) \in M^{2}$, which means that $(x, y) \sqsubseteq_{M^{2}}(a, b)$. Thus, $\sqsubseteq_{M^{2}}$ is transitive, that is, a preorder on $X^{2}$. The converse is similar.

(3) $[\Rightarrow]$ Suppose that $M^{2}$ is $(F, g)$-closed. Let $(x, y),(u, v) \in X^{2}$ be such that $G^{2}(x, y) \sqsubseteq_{M^{2}}$ $G^{2}(u, v)$, that is, $(g x, g y) \sqsubseteq_{M^{2}}(g u, g v)$, which means that either $(g x, g y)=(g u, g v)$ or $(g u, g v$, $g x, g y) \in M^{2}$. If $G^{2}(x, y)=G^{2}(u, v)$, then $T_{F}^{2}(x, y)=T_{F}^{2}(u, v)$ because $M^{2}$ is $(F, g)$-compatible. On the other case, if $(g u, g v, g x, g y) \in M^{2}$, then $(F(u, v), F(v, u), F(x, y), F(y, x)) \in M^{2}$ because $M^{2}$ is $(F, g)$-closed. This is equivalent to $(F(x, y), F(y, x)) \sqsubseteq_{M^{2}}(F(u, v), F(v, u))$, that is, $T_{F}^{2}(x, y) \sqsubseteq_{M^{2}} T_{F}^{2}(u, v)$. Therefore, $T_{F}^{2}$ is $\left(G^{2}, \sqsubseteq_{M^{2}}\right)$-nondecreasing.

$[\Leftarrow]$ Assume that $T_{F}^{2}$ is $\left(G^{2}, \sqsubseteq_{M^{2}}\right)$-nondecreasing and let $x, y, u, v \in X$ such that $(g x, g y$, $g u, g v) \in M$. In this case, $G^{2}(u, v)=(g u, g v) \sqsubseteq_{M^{2}}(g x, g y)=G^{2}(x, y)$. As $T_{F}^{2}$ is $\left(G^{2}, \sqsubseteq_{M^{2}}\right)$ nondecreasing, we have $T_{F}^{2}(u, v) \sqsubseteq_{M^{2}} T_{F}^{2}(x, y)$, which means that either $(F(x, y), F(y, x))=$ $(F(u, v), F(v, u))$ or $(F(x, y), F(y, x), F(u, v), F(v, u)) \in M$.

(4) It follows from the fact that $M$ is also $F$-closed.

\section{Corollary 40 Theorem 21 immediately follows from Theorem 33.}

Proof It follows from Lemma 39 and Corollary 35 considering the subset $M=\{((x, y, z)$, $\left.(u, v, w)) \in X^{6}: x \succcurlyeq u, y \preccurlyeq v, z \succcurlyeq w\right\}$ and taking into account the following facts:

- if $\varphi \in \Phi^{\prime}$ and if $\lambda>0$ is given, then $\varphi_{\lambda} \in \Phi^{\prime}$, where $\varphi_{\lambda}(t)=\lambda \varphi(t / \lambda)$ for all $t \geq 0$;

- when $d$ is a complete metric on $X$, the mapping $D^{3}$, defined by

$$
D^{3}((x, y, z),(u, v, w))=\frac{d(x, u)+d(y, v)+d(z, w)}{3} \quad \text { for all }(x, y, z),(u, v, w) \in X^{3},
$$

is a complete metric on $X^{3}$.

In the same way, the following result can be proved using the $(O, M)$-compatibility involved in Theorem 33.

\section{Corollary 41 Theorem 11 immediately follows from Theorem 33.}

\section{Remark 42}

1. Notice that the main results in [28] do not assume that $M$ verifies the transitive property. Therefore, they cannot be directly deduced from Theorem 33. However, they are consequences of Corollary 34 . 
2. Finally, we point out that, following well-known techniques (which can be found in

[7], Section 6), the unidimensional Theorem 33 can be particularized to the multidimensional case in which $X$ is replaced by $X^{n}$, and generating a large list of coupled, tripled and quadrupled possible results.

Example 43 Let $X=[0,1]$ provided with the Euclidean metric $d_{0}$ and let define the subset $M=X^{2}$, the mappings $T, g: X \rightarrow X$ and the function $\varphi:[0,+\infty) \rightarrow[0,+\infty)$ by

$$
T x=\frac{x}{4} \quad \text { and } \quad g x=\frac{x}{2} \quad \text { for all } x \in X ; \quad \varphi(t)=t / 2 \quad \text { for all } t \geq 0 .
$$

Since all conditions of Theorem 33 hold, then $T$ and $g$ has a coincidence point. In this case, $T$ and $g$ are continuous, and the only coincidence point between $T$ and $g$ is $x=0$.

Example 44 Let $X=[0,1]$ be provided with the Euclidean metric $d_{0}$ and let us define the subset $M \subset X^{2}$, the mappings $T, g: X \rightarrow X$, and the function $\varphi:[0,+\infty) \rightarrow[0,+\infty)$ by

$$
\begin{aligned}
& M=A \times A, \quad \text { where } A=\left\{\frac{1}{2^{n}}: n \in \mathbb{N}\right\} \cup\{0\} ; \quad \varphi(t)=t / 2 \quad \text { for all } t \geq 0 ; \\
& T x=\left\{\begin{array}{ll}
x / 4, & \text { if } x \in A, \\
0, & \text { if } x \in X \backslash A ;
\end{array} \quad g x= \begin{cases}x / 2, & \text { if } x \in A, \\
0, & \text { if } x \in X \backslash A .\end{cases} \right.
\end{aligned}
$$

If we take $x_{0}=1 \in A$, we have $\left(g x_{0}, T x_{0}\right)=(1 / 2,1 / 4) \in M$. Moreover, $T X=A \backslash\{1,1 / 2\} \subset$ $A \backslash\{1\}=g X$. If $(g x, g y) \in M$, we distinguish three cases.

- If $x, y \in A$, then

$$
\varphi(d(g x, g y))=\varphi\left(\left|\frac{x}{2}-\frac{y}{2}\right|\right)=\frac{1}{4}|x-y|=\left|\frac{x}{4}-\frac{y}{4}\right|=d(T x, T y) .
$$

- If $x \in A$ and $y \in X \backslash A$, then

$$
\varphi(d(g x, g y))=\varphi\left(\left|\frac{x}{2}-0\right|\right)=\frac{1}{4}|x-0|=\left|\frac{x}{4}-0\right|=d(T x, T y) .
$$

- If $x, y \in X \backslash A$, then $d(T x, T y)=0=\varphi(d(g x, g y))$.

Notice that $T$ and $g$ are not continuous on $X$, but $\left(X, d_{0}, M\right)$ is regular and $g X$ is closed. Since all conditions of Theorem 33 hold, then $T$ and $g$ has a coincidence point. In this case, all points in $X \backslash A$ are coincidence points between $T$ and $g$.

\section{Competing interests}

The authors declare that they have no competing interests.

\section{Authors' contributions}

All authors contributed equally to the writing of this paper. All authors read and approved the final manuscript.

\section{Author details}

'Department of Mathematics, Atilim University, Incek, Ankara 06836, Turkey. ${ }^{2}$ Nonlinear Analysis and Applied Mathematics Research Group (NAAM), King Abdulaziz University, Jeddah, Saudi Arabia. ${ }^{3}$ Department of Mathematics, University of Jaén, Campus las Lagunillas s/n, Jaén, 23071, Spain. ${ }^{4}$ Department of Mathematics, King Abdulaziz University, P.O. Box 80203, Jeddah, 21589, Saudi Arabia. ${ }^{5}$ Department of Mathematics and Statistics, Faculty of Science and Technology, Thammasat University Rangsit Center, Pathumthani, 12121, Thailand. 


\section{Acknowledgements}

This article was funded by the Deanship of Scientific Research (DSR), King Abdulaziz University, Jeddah. The third author, therefore, acknowledges with gratitude DSR for financial support. The second author has been partially supported by Junta de Andalucía by Project FQM-268 of the Andalusian CICYE.

Received: 7 December 2013 Accepted: 10 March 2014 Published: 08 Apr 2014

\section{References}

1. Gnana Bhaskar, T, Lakshmikantham, V: Fixed point theorems in partially ordered metric spaces and applications. Nonlinear Anal. 65(7), 1379-1393 (2006)

2. Berinde, $\mathrm{V}$, Borcut, $\mathrm{M}$ : Tripled fixed point theorems for contractive type mappings in partially ordered metric spaces. Nonlinear Anal. 74, 4889-4897 (2011)

3. Karapınar, E: Quartet fixed point for nonlinear contraction. http://arxiv.org/abs/1106.5472

4. Karapınar, E, Berinde, V: Quadruple fixed point theorems for nonlinear contractions in partially ordered metric spaces. Banach J. Math. Anal. 6, 74-89 (2012)

5. Roldán, A, Martínez-Moreno, J, Roldán, C: Multidimensional fixed point theorems in partially ordered complete metric spaces. J. Math. Anal. Appl. 396, 536-545 (2012)

6. Roldán, A, Martínez-Moreno, J, Roldán, C, Karapınar, E: Some remarks on multidimensional fixed point theorems. Fixed Point Theory (accepted)

7. Roldán, A, Karapınar, E: Some multidimensional fixed point theorems on partially preordered $G^{*}$-metric spaces under $(\psi, \varphi)$-contractivity conditions. Fixed Point Theory Appl. 2013, 158 (2013)

8. Wang, S: Coincidence point theorems for G-isotone mappings in partially ordered metric spaces. Fixed Point Theory Appl. 2013, 96 (2013)

9. Choudhury, BS, Kundu, A: A coupled coincidence point result in partially ordered metric spaces for compatible mappings. Nonlinear Anal. 73, 2524-2531 (2010)

10. Luong, NV, Thuan, NX: Coupled points in ordered generalized metric spaces and application to integro-differential equations. Comput. Math. Appl. 62(11), 4238-4248 (2011)

11. Hung, NM, Karapınar, E, Luong, NV: Coupled coincidence point theorem for O-compatible mappings via implicit relation. Abstr. Appl. Anal. 2012, Article ID 796964 (2012)

12. Ran, ACM, Reurings, MCB: A fixed point theorem in partially ordered sets and some applications to matrix equations. Proc. Am. Math. Soc. 132, 1435-1443 (2004)

13. Nieto, JJ, Rodríguez-López, R: Contractive mapping theorem in partially ordered sets and applications to ordinary differential equations. Order 22, 223-239 (2005)

14. Boyd, DW, Wong, JSW: On nonlinear contractions. Proc. Am. Math. Soc. 20, 458-464 (1969)

15. Mukherjea, A: Contractions and completely continuous mappings. Nonlinear Anal. 1(3), 235-247 (1997)

16. Romaguera, S: Fixed point theorems for generalized contractions on partial metric spaces. Topol. Appl. 159(1), 194-199 (2012)

17. Lakshmikantham, V, Ćirić, LJ: Coupled fixed point theorems for nonlinear contractions in partially ordered metric spaces. Nonlinear Anal. 70(12), 4341-4349 (2009)

18. Guo, D, Lakshmikantham, V: Coupled fixed points of nonlinear operators with applications. Nonlinear Anal. 11 623-632 (1987)

19. Samet, B, Vetro, C: Coupled fixed point F-invariant set and fixed point of N-order. Ann. Funct. Anal. 1, 46-56 (2010)

20. Sintunavarat, W, Kumam, P, Cho, YJ: Coupled fixed point theorems for nonlinear contractions without mixed monotone property. Fixed Point Theory Appl. 2012, 170 (2012)

21. Samet, B, Karapınar, E, Aydi, H, Rajic, C: Discussion on some coupled fixed point theorems. Fixed Point Theory Appl. 2013, 50 (2013)

22. Agarwal, R, Karapınar, E: Remarks on some coupled fixed point theorems in G-metric spaces. Fixed Point Theory Appl. 2013, 2 (2013)

23. Ding, H-S, Karapınar, E: A note on some coupled fixed point theorems on G-metric space. J. Inequal. Appl. 2012, 170 (2012)

24. Karapınar, E, Kaymakcalan, B, Tas, K: On coupled fixed point theorems on partially ordered G-metric spaces. J. Inequal. Appl. 2012, 200 (2012)

25. Karapınar, E, Roldán, A: A note on 'n-tuplet fixed point theorems for contractive type mappings in partially ordered metric spaces.' J. Inequal. Appl. 2013, 567 (2013)

26. Batra, R, Vashistha, S: Coupled coincidence point theorems for nonlinear contractions under $(F, g)$-invariant set in cone metric spaces. J. Nonlinear Sci. Appl. 6, 86-96 (2013)

27. Charoensawan, $\mathrm{P}$ : Tripled coincidence point theorems for a $\varphi$-contractive mapping in a complete metric space without the mixed g-monotone property. Fixed Point Theory Appl. 2013, 252 (2013)

28. Kutbi, MA, Roldán, A, Sintunavarat, W, Martínez-Moreno, J, Roldán, C: F-closed sets and coupled fixed point theorems without the mixed monotone property. Fixed Point Theory Appl. 2013, 330 (2013)

10.1186/1687-1812-2014-92

Cite this article as: Karapınar et al.: Discussion of coupled and tripled coincidence point theorems for $\varphi$-contractive mappings without the mixed g-monotone property. Fixed Point Theory and Applications 2014, 2014:92 